\title{
Avaliação de duas doses de propofol para infusão contínua em pombos domésticos
}

\author{
Evaluation of two intraosseous constant rate infusions of propofol in domestic pigeons \\ Luciana Dambrósio Guimarães', Cláudio Corrêa Natalini², Fabíola Niederauer Flores ${ }^{3}$, \\ Sheila Francheska Camargo ${ }^{4}$, Simone Bopp ${ }^{5}$ \& Ney Luis Pippi ${ }^{6}$
}

\begin{abstract}
RESUMO
O uso do propofol em aves tem sido pesquisado e parece ser uma alternativa viável à anestesia inalatória em algumas situações. Este estudo tem como objetivo avaliar os efeitos de duas doses de propofol para infusão contínua, administrado pela via intra-óssea, em pombos domésticos. Sete animais foram anestesiados em diferentes ocasiões. Inicialmente, a anestesia foi realizada com isofluorano em oxigênio, para introdução de uma agulha intra-óssea (acesso intra-ósseo). Após a recuperação, os animais foram anestesiados com propofol na dose necessária para permitir a intubação endotraqueal. Os pombos foram conectados a um sistema não reinalatório com fluxo de $\mathrm{O}_{2}$ de $500 \mathrm{~mL} / \mathrm{min}$ e a anestesia foi mantida com $1 \mathrm{mg} / \mathrm{kg} / \mathrm{min}$ (dose baixa) ou $3 \mathrm{mg} /$ $\mathrm{kg} / \mathrm{min}$ (dose alta) de propofol por 30 minutos. Frequiência cardíaca, frequiência respiratória, pressão arterial sistólica indireta, temperatura cloacal, oximetria de pulso e gases sangüíneos arterial foram mensurados antes da indução da anestesia e aos 15 e 30 minutos do período de manutenção. A dose necessária para indução anestésica foi de 14.49 $\pm 2,91 \mathrm{mg} / \mathrm{kg}$. A dose baixa de propofol produziu acidose respiratória e hipotensão. Ocorreram movimentos espontâneos e cinco dos sete animais foram extubados devido a anestesia superficial. A dose alta de propofol resultou em hipotensão, hipotermia e apnéia, necessitando de ventilação artificial. Devido ao uso da ventilação manual, a dose alta de propofol produziu valores mais altos de $\mathrm{pH}$ e mais baixos de $\mathrm{PaCO}_{2}$. O tempo de extubação com a dose baixa foi de $16,33 \pm 12,42$ minutos $(n=2)$ e de $52,33 \pm 22,37$ minutos $(n=7)$ com a dose alta. Baseado nestes resultados recomenda-se que o propofol seja utilizado com cautela em pombos domésticos.
\end{abstract}

Descritores: anestesia, aves, infusão contínua.

\section{ABSTRACT}

The use of propofol has been investigated in birds and appears to be a viable alternative to inhalant anesthesia in some instances. The present study evaluated the effects of two constant rate infusions of propofol, administered by the intraosseous route, in domestic pigeons. Seven domestic pigeons were anesthetized on two different occasions. Firstly, animals were anesthetized with isoflurane in oxygen for the introduction of a $30 \mathrm{~mm}$ long, 8 Gauge needle into the ulna (intraosseous access). After recovery, anesthesia was reinduced with propofol, at a dose sufficient to allow endotracheal intubation. Pigeons connected to a non rebreathing circuit and anesthesia was maintained with either $1 \mathrm{mg} / \mathrm{kg} / \mathrm{min}$ (low-dose propofol) or $3 \mathrm{mg} / \mathrm{kg} / \mathrm{min}$ (highdose propofol) for a further 30 minutes. Heart rate, respiratory rate, indirect systolic blood pressure, cloacal temperature, pulseoximetry, and arterial blood gases were measured before the anesthetic induction and at 15 and 30 minutes during the maintenance period. The dose of propofol for induction of anesthesia was $14.49 \pm 2,91 \mathrm{mg} / \mathrm{kg}$. The low-dose propofol resulted in respiratory acidosis and hypotension. Spontaneous movements were present, and 5/7 pigeons were extubated due to superficial anesthesia. The high-dose propofol induced hypotension, hypothermia and apnea requiring artificial ventilation. Due to the use of manual ventilation, high-dose propofol resulted in significantly higher $\mathrm{pH}$ and lower $\mathrm{PaCO} 2$ values. The time for extubation from propofol anesthesia was 16,33 $\pm 12,42$ minutos $(n=2)$ with low-dose and $52,33 \pm 22,37$ minutos $(n=7)$ with high-dose propofol. Due to the side effects observed in this study, we recommend that propofol be used with caution in domestic pigeons.

Key words: anesthesia, birds, constant rate infusions.

${ }^{1}$ Av. Fernando Correa da Costa s/n. Departamento de Clínica Médica Veterinária, Universidade Federal de Mato Grosso. Cep: $78080-900$. ${ }^{2}$ Centro de Pesquisas Experimentação Animal. Hospital de Clínicas de Porto Alegre, RS. ${ }^{3}$ Universidade do Estado de Santa Catarina (UDESC). ${ }^{4}$ Médica Veterinária Autônoma. ${ }^{5}$ Faculdade Integrado de Campo Mourão. ${ }^{6}$ Universidade Federal de Santa Maria (UFSM). CORRESPONDÊNCIA: L.D. Guimarães [1dguimaraes@ufmt.br ; Fax: 653615 8614]. 


\section{INTRODUÇÃO}

O uso de anestésicos voláteis em aves apresenta desvantagens em algumas situações, como doenças graves do trato respiratório, reparação de fraturas em ossos pneumáticos e cirurgias do trato respiratório ou abdominal, com invasão dos sacos aéreos [3,5,9].

A friabilidade dos vasos e o temperamento do paciente dificultam a utilização da via intravenosa (IV) em algumas espécies de aves, assim muitos anestésicos são administrados pela via intra-muscular (IM). O acesso intra-ósseo foi adaptado de técnicas utilizadas na pediatria humana e pode ser utilizado como uma alternativa à cateterização IV [6-8,10,11].

O propofol é um agente anestésico não barbitúrico muito utilizado em humanos e animais domésticos, administrado pela via IV na forma de bolus ou infusão contínua. As vantagens são indução e recuperação rápida, meia-vida de eliminação curta e toxicidade limitada [4]. Em aves, a margem de segurança é estreita para a indução da anestesia em pombos domésticos, os efeitos cardiovasculares foram significativos durante a manutenção anestésica em galinhas, mas foi considerado seguro em uma coruja submetida à ressecção traqueal [1,5].

Nenhum anestésico injetável de curta ação é considerado seguro em aves [3]. As pesquisas sobre a utilização do propofol ainda são poucas e é necessário determinar diferenças entre as espécies e a segurança do fármaco. O objetivo deste estudo é avaliar os efeitos de duas doses de propofol para a manutenção da anestesia através de infusão contínua, administrado pela via intra-óssea, em pombos domésticos. A hipótese é que uma das doses de propofol utilizadas possa ser considerada segura para anestesia em pombos domésticos.

\section{MATERIAIS E MÉTODOS}

Para este estudo foram utilizados sete pombos

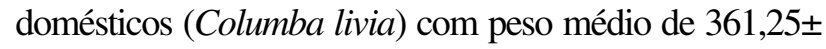
$44,46 \mathrm{~g}$, considerados clinicamente saudáveis através de exame clinico. Os animais foram submetidos a um período de adaptação de, no mínimo, 20 dias em uma sala com ventilação e luminosidade apropriada, contendo poleiros, e receberam ração farelada para aves acrescida de vermífugo e água ad libidum. Após o período de adaptação os mesmos foram anestesiados em duas ocasiões, com tratamentos diferenciados, em intervalo de pelo menos quatro dias.
Os animais foram anestesiados com $3 \mathrm{~V} \%$ de isofluorano em oxigênio $(500 \mathrm{~mL} / \mathrm{min}$.) para a remoção manual das penas e a realização da técnica de acesso intra-ósseo. A região do carpo foi submetida à anti-sepsia com álcool para introdução de uma agulha hipodérmia $30 \times 8$ no centro da porção distal da ulna, paralela ao plano médio do osso. O bizel da agulha perfurou a cortical óssea através de pressão e movimento leve de rotação e, após a perda da resistência, a agulha foi introduzida em todo o seu comprimento no interior do canal medular.

Após a recuperação da anestesia inalatória, os animais foram re-induzidos com propofol ${ }^{1}$ na dose necessária para permitir a intubação endotraqueal. A seguir, os mesmos foram intubados com sonda de Murphy número 2 ou 2,5 e conectados a um sistema não reinalatório (sistema de Bain) com fluxo de $\mathrm{O}_{2}$ de $500 \mathrm{~mL} / \mathrm{min}$. A anestesia foi mantida com $1 \mathrm{mg} / \mathrm{kg} / \mathrm{min}$ (dose baixa) ou $3 \mathrm{mg} / \mathrm{kg} / \mathrm{min}$ de propofol (dose alta) durante 30 minutos, com os animais em decúbito dorsal sobre bolsas de água quente (aquecidas até $40^{\circ}$ ). O propofol foi diluído em solução fisiológica até concentração suficiente para alcançar um volume de administração de $0,1 \mathrm{~mL} / \mathrm{min}$ que foi administrado de maneira manual a cada minuto.

Frequiência cardíaca (FC), freqüência respiratória $(f)$, pressão arterial sistólica indireta (PAS), temperatura cloacal $(\mathrm{T})$, oximetria de pulso $\left(\mathrm{SpO}_{2}\right)$ e gases sanguiíneos arterial foram mensurados antes da indução da anestesia e aos 15 e 30 minutos do período de manutenção. Após o término da infusão foi avaliado o tempo decorrido até a extubação dos animais.

A FC e $\mathrm{SpO}_{2}$ foram avaliadas através de oximetria de pulso $^{2}$ com o sensor do equipamento nos dígitos dos animais; a $f$ pelos movimentos do esterno; a PAS através de Doppler ultrassônico ${ }^{3}$ com sensor e manguito sobre leito arterial localizado na perna dos animais, e os gases sanguiíneos foram mensurados através de analisador de gases sanguíneos ${ }^{4}$ à $37^{\circ} \mathrm{C}$. $\mathrm{O}$ sangue arterial foi colhido através de punção percutânea das artérias radial, ulnar ou braquial ou cateterização da artéria ulnar, num volume de $0,4 \mathrm{~mL}$, com seringa de insulina previamente heparinizada.

Os dados foram avaliados através de análise de variância (ANOVA) e teste de Tukey para comparação entre os tempos e os grupos e apresentados como média \pm desvio padrão. 


\section{RESULTADOS}

A dose de propofol para indução anestésica foi de $14,49 \pm 2,91 \mathrm{mg} / \mathrm{kg}$.

A dose baixa de propofol $(1 \mathrm{mg} / \mathrm{kg} / \mathrm{min})$ produziu valores inferiores aos obtidos antes da anestesia a partir dos 15 minutos do período de manutenção na PAS (Tabela 1) e no $\mathrm{pH}$ e valores superiores na $\mathrm{PaCO}_{2}$ (Tabela 2). A T reduziu somente aos 30 minutos (Tabela 1). Ocorreram movimentos espontâneos e cinco dos sete animais foram extubados durante o período de manutenção. O tempo de extubação do restante dos animais $(n=2)$ foi de $16,33 \pm 12,42$ minutos.
A dose alta de propofol ( $3 \mathrm{mg} / \mathrm{kg} / \mathrm{min})$ resultou em redução da PAS, T (Tabela 1), apnéia duradoura, que fez necessário o uso de ventilação artificial, valores mais altos de $\mathrm{pH}$ e mais baixos de $\mathrm{PaCO}_{2}$ a partir dos 15 minutos, quando comparados aos valores obtidos antes da anestesia (Tabela 2). Os animais foram ventilados de maneira manual mantendo a $f$ em seis movimentos respiratórios/minuto. O tempo de extubação foi de $52,33 \pm 22,37$ minutos.

Foi observada diferença significativa entre as doses no $\mathrm{pH}$ e $\mathrm{PaCO}_{2}$ a partir dos 15 minutos de anestesia (Tabela 2).

Tabela 1. Variáveis relacionadas ao sistema cardiovascular e respiratório de pombos domésticos submetidos a infusão contínua com dose baixa $(1 \mathrm{mg} / \mathrm{kg} / \mathrm{min})$ e alta $(3 \mathrm{mg} / \mathrm{kg} / \mathrm{min})$ de propofol, administrado pela via intra-óssea. Valores obtidos antes da anestesia e aos 15 e 30 minutos do período de manutenção anestésica.

\begin{tabular}{lcccc}
\hline Variável & Dose & Antes anestesia & 15 minutos & 30 minutos \\
\hline \multirow{2}{*}{ Freqüência cardíaca (batimentos/ min.) } & Baixa & $146,33 \pm 20,44$ & $192,83 \pm 52,81$ & $186,33 \pm 41,15$ \\
& Alta & $155,00 \pm 23,40$ & $181,14 \pm 65,19$ & $178,57 \pm 58,43$ \\
\hline \multirow{2}{*}{ Freqüência respiratória (movimentos/min.) } & Baixa & $35,67 \pm 5,99$ & $38,80 \pm 4,24$ & $24,00 \pm 10,20^{*}$ \\
& Alta & $28,00 \pm 9,56$ & $06,00 \pm 0,00$ & $06,00 \pm 0,00$ \\
\hline \multirow{2}{*}{ Saturação de oxigênio na hemoglobina (\%) } & Baixa & $092,83 \pm 3,18$ & $100,00 \pm 0,00$ & $099,20 \pm 0,84$ \\
& Alta & $096,83 \pm 02,86$ & $099,86 \pm 0,38$ & $099,33 \pm 0,52$ \\
\hline \multirow{2}{*}{ Pressão arterial sistólica (mmHg) } & Baixa & $124,67 \pm 16,91$ & $080,67 \pm 26,91^{*}$ & $063,83 \pm 29,92^{*}$ \\
& Alta & $127,00 \pm 48,92$ & $074,86 \pm 27,90^{*}$ & $058,67 \pm 23,58^{*}$ \\
\hline Temperatura cloacal $\left({ }^{\circ} \mathrm{C}\right)$ & Baixa & $041,28 \pm 0,56$ & $040,23 \pm 1,14$ & $039,77 \pm 1,14^{*}$ \\
\hline
\end{tabular}

*variação significativa em relação aos valores obtidos antes da anestesia $(\mathrm{p}<0,05)$.

\begin{tabular}{|c|c|c|c|c|}
\hline Variável & Dose & Antes anestesia & 15 minutos & 30 minutos \\
\hline \multirow{2}{*}{$\mathrm{pH}$} & Baixa & $07,43 \pm 0,07$ & $07,32 \pm 0,09^{*}+$ & $07,31 \pm 0,05^{\star}+$ \\
\hline & Alta & $07,45 \pm 0,02$ & $07,52 \pm 0,11+$ & $07,55 \pm 0,06+$ \\
\hline \multirow{2}{*}{$\mathrm{PaCO}_{2}$} & Baixa & $33,72 \pm 3,64$ & $50,94 \pm 14,13^{*}+$ & $53,83 \pm 7,12^{*}+$ \\
\hline & Alta & $34,65 \pm 2,43$ & $30,58 \pm 11,43+$ & $25,01 \pm 7,09+$ \\
\hline \multirow{2}{*}{$\mathrm{HCO}_{3}$} & Baixa & $021,7 \pm 2,10$ & $24,25 \pm 2,33$ & $25,90 \pm 1,69^{*}+$ \\
\hline & Alta & $22,91 \pm 2,53$ & $22,84 \pm 4,10$ & $20,37 \pm 3,17+$ \\
\hline
\end{tabular}




\section{DISCUSSÃO}

A dose média de propofol utilizada para a indução da anestesia $(14 \mathrm{mg} / \mathrm{kg})$ pela via intra-óssea foi idêntica à utilizada como adjuvante da anestesia com cetamina pela via IV e que causou uma potente depressão respiratória em pombos [1].

A técnica de acesso intra-ósseo é prática, de fácil realização, eficiente e a administração de anestésicos não provoca danos persistentes à cartilagem ulnar ou medula óssea. As substâncias injetadas no espaço intra-ósseo são absorvidas por sinusóides que drenam para os canais venosos medulares e chegam até a circulação sistêmica. A velocidade de entrada destas substâncias na circulação é muito rápida, em mamíferos [8]. Pode-se extrapolar esses dados para aves, o que justifica os rápidos efeitos observados na indução da anestesia e a dose idêntica a utilizada pela via IV em pombos domésticos. É necessário uma única punção óssea para evitar o extravasamento do fármaco por outros orifícios, o que dificulta a indução anestésica [11].

Inicialmente, tentou-se fazer a administração do anestésico através de uma bomba de infusão de seringa ${ }^{5}$, porém, com freqüência a bomba apresentava oclusão. Existem as seguintes justificativas para este fato: lenta transferência do fármaco do espaço intra-ósseo para o sangue, volume de fluido administrado suficiente para encher o canal medular (mantendo uma pressão intra-óssea para parar a infusão) e agulha de diâmetro pequeno (impondo resistência para parar a infusão). A interferência do diâmetro da agulha deveria ocorrer quando a bomba de infusão foi testada sem o acesso intra-ósseo, o que não ocorreu. A bomba de infusão pode administrar o volume de 0,1 mL lentamente, em um minuto, de maneira que o mesmo seria absorvido e, assim, não permaneceria no canal medular quantidade suficiente para impedir a infusão. A administração manual impõe maior pressão, provavelmente, forçando a transferência do líquido do canal medular para os sinusóides, tornando a velocidade de entrada do propofol na circulação sistêmica mais rápida. Em mamíferos, a velocidade de administração de líquidos pela via intra-óssea é limitada a $11 \mathrm{ml} / \mathrm{min}$ com fluxo dependente da gravidade e $24 \mathrm{ml} /$ min com fluxo sobre pressão de $300 \mathrm{mmHg}$ [6]. Velocidade de fluxo para esta via não foi relatada em aves. Assim, optou-se por administrar o fármaco de maneira manual injetando-se com uma seringa $0,1 \mathrm{~mL}$ da diluição do anestésico a cada minuto.
Os pombos apresentaram reflexo palpebral espontâneo, movimentos da terceira pálpebra e movimentos espontâneos ou propositais em resposta à manipulação quando foi administrada a dose baixa de propofol. Cinco dos setes animais necessitaram de extubação devido ao plano anestésico muito superficial. Apesar da anestesia superficial, os animais apresentaram hipotensão e acidose respiratória já a partir dos 15 minutos de anestesia. Isso não foi observado em patos, em plano leve de anestesia, pois a pressão arterial média foi mantida dentro da normalidade [4].

A dose alta de propofol produziu hipotensão e apnéia. A ventilação manual foi instituída através de compressão do balão reservatório do aparelho de anestesia e observação da amplitude da parede costal dos animais, numa freqüência de seis movimentos respiratórios por minuto. A ventilação artificial foi eficiente e manteve os valores de $\mathrm{pH}$ e $\mathrm{PaCO}_{2}$ próximos aos valores obtidos antes da anestesia, o que resultou numa diferença estatística significativa entre as doses utilizadas.

O propofol produz depressão cardiovascular e respiratória dose-dependente em mamíferos e aves [4,9]. A hipoventilação pode ser corrigida pela ventilação mecânica ou assistida, o que nem sempre é possível em aves muito pequenas [3]. Acredita-se que neste estudo a apnéia tenha ocorrido por fator dosedependente, pois imediatamente após a indução anestésica, idêntica em ambos os tratamentos, a presença da mesma não foi observada.

A hipotermia ocorreu com ambas as doses, com maior intensidade quando a mais alta foi utilizada. A redução da temperatura corporal é dose-dependente e relativamente liniar com o tempo. A manutenção da temperatura corporal é de extrema importância em aves, pois a hipotermia pode causar prolongamento no tempo de recuperação, depressão do sistema respiratório e morte [10].

Recuperação prolongada foi observada com a dose mais alta, provavelmente devido à hipotermia, já que a principal vantagem do propofol é a rápida recuperação. O tempo observado até a estação foi de $11 \pm 6$ minutos em perus selvagens e $23 \pm 17$ minutos em pombos após uma dose de indução [1,9].

As doses de indução e manutenção da anestesia utilizadas neste estudo foram superiores às de outras aves, tais como perus selvagens (Meleagris gallipavo), galinhas (Gallus gallus domesticus), patos (Anãs platyrhynchos), avestruz (Struchio camelus) e 
coruja (Tyto Alba), pela via IV [2-5,9]. A anestesia foi caracterizada por indução rápida e suave, bom relaxamento muscular, recuperação rápida, depressão respiratória e cardiovascular significativa em todas as espécies citadas, com exceção de um único exemplar de coruja de celeiro onde foi considerada segura [3-5,7,9].

É sabido que existe uma grande variação nas doses e efeitos dos fármacos nas diferentes espécies de aves e por isso há a necessidade de estudos para determinar essas variações. Além disso, poderiam ser elaboradas técnicas de anestesia balanceada, o que minimizaria os efeitos colaterais dos fármacos. A anestesia balanceada é muito utilizada em humanos e animais domésticos e poder-se-ia extrapolar essas técnicas para aves. Dessa maneira o anestesista seria orientado na escolha do melhor protocolo anestésico a ser utilizado em uma ampla variedade de espécies, tornando a anestesia de aves cada vez mais segura.

\section{CONCLUSÕES}

Baseado nos resultados desse estudo recomenda-se que a infusão contínua de propofol para manutenção da anestesia seja utilizada com cautela em pombos domésticos. Técnicas de anestesia balanceada devem ser estudas para minimizar os efeitos colaterais produzidos pelos anestésicos.

\section{NOTAS INFORMATIVAS}

${ }^{1}$ Diprivan ${ }^{\circledR}$ - Cristália - Itapira, SP/Brasil.

${ }^{2}$ Modelo Nellcor N - 200 - Nellcor - São Paulo, SP/Brasil. ${ }^{3}$ Modelo 841 - A - Parks Medicals Eletronics Inc. - Aloha, Oregon/USA.

${ }^{4}$ Modelo AVL 990-S - Biomedical Instrumentals - Áustria. ${ }^{5}$ Modelo ST 680 - SAMTRONIC - São Paulo, SP/Brasil.

\section{REFERÊNCIAS}

1 Fitzgerald G. \& Cooper J.E. 1990. Preliminary studies on the use of propofol in the domestic pigeon (Columba livia). Research in Veterinary Science. 49: 334-338.

2 Langan J.N., Ramsay E.C. \& Blackford J.T. 2000. Cardiopulmonary and sedative effects of intramuscular medetomidineketamine and intravenous propofol in ostriches (Struthio camelus). Journal of Avian Medicine and Surgery. 14: 2-7.

3 Lukasik V.M. Gentz E.J., Erb H.N., Ludders J.W. \& Scarlett J.M. 1997. Cardiopulmonary effects of propofol anesthesia in chickens (Gallus gallus domesticus). Journal of Avian Medicine and Surgery. 11: 93-97.

4 Machin K. \& Caulkett N.A. 2000. Evaluation of isoflurano and propofol anesthesia for intraabdominal trasmitter placement in nesting female canvasback ducks. Journal of Wildlife Diseases. 36: 324-334.

5 Mama K.R., Phillips L.G. \& Pascoe P.J. 1996. Use of propofol foi induction and maintenance of anesthesia in a barn owl (Tyto alba) undergoing tracheal resection. Journal of Zoo and Wildlife Medicine. 27: 397-401.

6 Otto C.M., Kaufman G.M. \& Crowe D.T. Intraosseous infusion of fluids and therapeutics. Compedium on Continuing Education: Small Animal Practice. 11: 421-431.

7 Paul-Murphy J. \& Fialkowski J. 2001. Injectable anesthesia and analgesia of birds. Disponível em: <http://www.vetmed.Imu.de/ chir_k/student/downloads/injectableanesthesiabiedesivis.pdf>. Acesso em: 5 de agosto de 2001.

8 Ritchie B.W., Otto C.M., Latimer K.S. \& Crowe D.T. 1990. A technique of intraosseous cannulation for intravenous therapy in birds. Compedium on Continuing Education: Small Animal Practice. 12: 55-58.

9 Schumacher J., Citino S.B., Hernandez K. \& Brandon D. 1997. Cardiopulmonary and anesthetic effects of propofol in wild turkeys. American Journal Veterinary Research. 58: 1014-1017.

10 Skarda R.T., Berdnarski R.M. \& Muir W.W. 1995. Anesthesia procedures in exotic pets. In: Handbook of Veterinary Anesthesia. 2nd edn. St. Louis: Mosby, pp. 341-371.

11 Valverde A., Bienzle D., Smith D.A., Dyson D.H. \& Valliant A.E. 1993. Intraosseous cannulation and drug administration for induction of anesthesia in chickens. Veterinary Surgery. 22: 240-244.

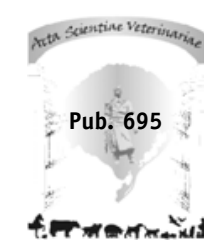

